\title{
Breast findings incidentally detected on body MRI
}

\author{
Bianca Bignotti ${ }^{\text {* }}$, Giulia Succio ${ }^{2}$, Francesca Nosenzo ${ }^{1}$, Michela Perinetti ${ }^{1}$, Licia Gristina ${ }^{1}$, Stella Barbagallo', \\ Lucia Secondini ${ }^{1}$, Massimo Calabrese ${ }^{2}$ and Alberto Tagliafico ${ }^{3}$
}

\begin{abstract}
Objectives: To evaluate breast findings incidentally detected on body MRI.

Methods: A retrospective review of the institutional database identified 1752 body MRI performed between January 2015 and September 2015. MRI of women with breast tissue visible in the field-of-view were reviewed for breast findings. Breast findings were classified with the breast imaging reporting and data system (BI-RADS) lexicon. The standard statistic, costs of additional work-up, and the clinical relevance were used to describe breast findings, and we calculated $95 \%$ exact confidence intervals (Cls).

Results: 440 body MRI of 440 women (mean age: $57 \pm 20$ years) included breast tissue in the field-of-view. A total of 41 breast findings were identified in 41 patients. Breast findings were classified BI-RADS $2 \mathrm{~N}=25, \mathrm{BI}$-RADS $3 \mathrm{~N}=13$, BI-RADS $4 \mathrm{~N}=3$. A total of $3.6 \%$ [95 \% Cl 1.6\%, 5.6\%] women with breast tissue visible on MRI had a recommendation for further imaging work-up for a breast finding. The $18.7 \%$ (3 of 16) of these patients had a clinically important finding (breast cancer). Further imaging evaluation increased costs of $€ 108.3$ per patient with a breast finding.
\end{abstract}

Conclusions: Clinically important breast findings could be detected on body MRI in up to $0.7 \%$ (3 of 440) of women.

Keywords: Breast, Magnetic resonance imaging, Body, Cancer, Costs

\section{Background}

Body magnetic resonance imaging (MRI) is a highly sensitive imaging method to assess morphological structures and the presence of a lesion (Schmidt et al. 2013). Since there has been an increased use of MRI in clinical practice, an increased number of incidental findings are expected (Chernyak et al. 2015; Wagner and Aron 2012; Sebastian et al. 2013; Heller et al. 2013; Khosa et al. 2013; Patel et al. 2013). Indeed, incidental findings are increasingly common, and the American College of Radiology developed guidelines for management of incidental findings detected during abdominal and pelvic MRI or computed tomography (CT) (Sebastian et al. 2013; Heller et al. 2013; Khosa et al. 2013; Patel et al. 2013). In addition, several studies evaluated incidental findings outside the region-of-interest during MRI of the heart, breast

\footnotetext{
*Correspondence: bignottibianca@gmail.com

1 Department of Health Sciences (DISSAL), University of Genova, Via A. Pastore 1, 16132 Genoa, Italy

Full list of author information is available at the end of the article
}

and spine, using an expanded field-of-view (FOV) (Wyttenbach et al. 2012; Bazzocchi et al. 2012; Dewey et al. 2007; Maxwell et al. 2015). Regarding MRI of the abdomen and the chest (body MRI), different field-of-views are used, based on the clinical suspicious and the specific region-of-interest to be evaluated. In the most frequent clinical indications of body MRI, the anteroposterior size of FOV is approximately 30 centimetres (Erturk et al. 2009; Chang et al. 2008; Lee et al. 2014). Therefore, some breast tissue could be included in body MR examinations, and consequently, some incidental breast findings could be potentially detected. In literature, incidental breast findings have been evaluated and described on $\mathrm{CT}$ and (18) F-fluorodeoxyglucose (FDG) positron emission tomography (PET)-CT (Bach et al. 2013; Monzawa et al. 2013; Benveniste et al. 2015). In a population of patients with known non-mammary malignancies, $6 \%$ of incidental breast lesions detected on FDG PET/CT were primary breast cancer (Benveniste et al. 2015). Several studies evaluated potentially relevant incidental findings 
on MRI, including breast findings, but all of these studies dealt with whole-body MRI and not with more tailored MR examinations (Cieszanowski et al. 2014; Tarnoki et al. 2015). To the best of our knowledge, there are no studies in literature dealing with breast findings detected on body (abdominal and chest) MRI. Therefore, the aim of our study was to evaluate breast findings incidentally detected on body MRI, recommendations for further imaging work-up, and the clinical relevance and health care costs.

\section{Methods}

This study was notified to the Institutional Review Board, and the requirement for informed consent was waived. Our Centre is a Tertiary Centre with more than 6.000 MRI examinations performed in a year and more than 2.000 are body MRI examinations. Figure 1 shows the flow-chart of the study.

A retrospective query of our body MRI database was performed by seven radiologists in March 2016. The seven radiologists (B.B., G.S., F.N., M.P., L.G., S.B., L.S.) had more than 3 years of experience in body and breast imaging. The retrospective query included the review of the images of body MRI with breast tissue visible on abdominal and chest MRI. The query was done between January 2015 and September 2015. At least 6-months follow-up period of the lesions classified as benign was warranted. For patients who underwent more than one body

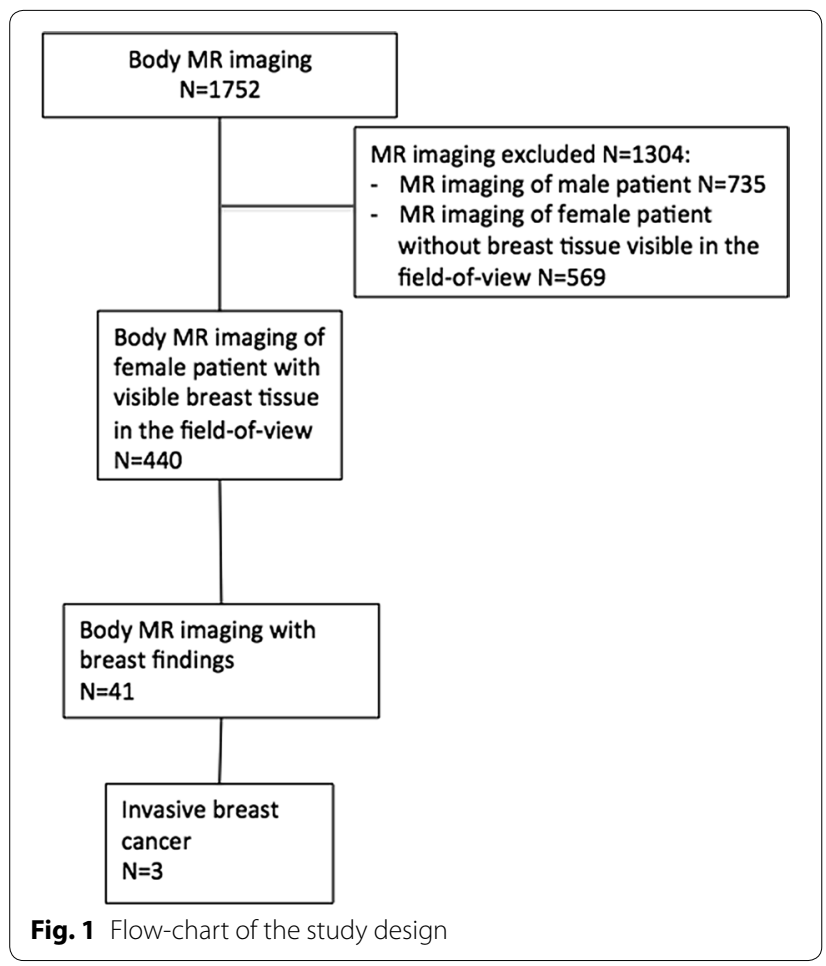

MRI during the time interval, we recorded each MRI as a separate event. Dedicated breast, musculoskeletal and MR neuro imaging examinations were excluded from the investigation.

A total of 1752 body MRI examinations were reviewed. MRI examinations of male patients and with no breast tissue visible were excluded. Breast findings were evaluated on the remaining body MRI in female patients with breast tissue visible.

For each body MRI examination with breast tissue visible, the percentage of breast tissue visible was recorded and visually estimated as more or less than $50 \%$ of the total breast volume. In addition, patient age, magnetic field where the MRI was performed and MRI type (abdominal or chest) were recorded. In particular, the FOV size and the type of the contrast agent used and the presence or absence of diffusion-weighted images were recorded.

\section{MRI technique and image interpretation}

Abdominal and chest MRI were performed with the patient in a supine position by using a $1.5 \mathrm{~T}$ (Magnetom Avanto, Siemens, Germany) or a 3.0 T (GE Signa HDx 3.0T, General Electric Medical Systems, Milwaukee, WI, USA) scanners with dedicated phased-array coil usually in combination with table-embedded spine coils. We performed abdominal and chest MRI following Institutional guidelines. Each examination was tailored to the patient clinical feature (body mass index) and the body region to be studied.

\section{Image interpretation}

Two breast radiologists (A.T., M.C.), with more than 5 and 10 years of experience in breast MRI respectively, reviewed the MR images with detected breast findings and recorded the characteristic of the breast findings following the breast imaging reporting and data system (BI-RADS) MRI lexicon (American college of radiology breast imaging reporting and data system (BI-RADS) Atlas 2013). The two radiologists were blinded to clinical outcomes and previous breast imaging evaluation. For any patient who had more than one breast finding, each finding was included in the study. A single breast finding was considered for any patient with more than one simple breast cyst. For each body MRI with a breast finding, one radiologist (B.B.) reviewed the MRI report to evaluate any recommendations for additional work-up. The same radiologist assessed in the electronic medical record the availability of clinical follow-up and/or previous MRI examinations for comparison, or results of any additional imaging and histopathological work-up at our Institution for at least 6 months after the body MRI to evaluate the clinical relevance of breast finding and to 
follow-up the lesion classified as benign. Any breast findings initially assigned to BI-RADS category 3 but previously evaluated and described in the electronic medical record was downgraded and assigned to BI-RADS category 2 , as previously performed in other similar setting (Niell et al. 2015).

\section{Cost analysis}

The costs of further imaging evaluation and procedures were calculated on the basis of the reimbursement proposed at our Institution ( $€ 120$ for standard mammography; $€ 120$ for breast ultrasonography; $€ 400$ for breast MRI; $€ 200$ for ultrasonography breast core-needle biopsy; $€ 200$ for ultrasound-guided preoperative lesion marking) and were intended as costs theoretically paid by patients. All further breast imaging work-up, follow-up and procedures performed after body MRI recommendation was included in the cost analysis.

\section{Statistical analysis}

A descriptive analysis was performed among the patient population and corresponding MRI characteristic included in the study (patient age, percentage of breast tissue visible, MR magnetic field, type of MRI (abdominal or chest), the FOV size, the type of the contrastmedia, number of examinations with diffusion-weighted images). We compared the presence of a breast finding and subsequent recommendations for further imaging and procedures between the groups of patients that performed abdominal and chest MRI, between the groups of patients that performed contrast-enhanced MRI and non-contrast enhanced MRI using the Fisher's exact test. A $\mathrm{p}$ value of less than 0.05 was considered statistically significant significance (MedCalc Software bvba. Acacialaan 22, Ostend, Belgium) and we calculated $95 \%$ exact confidence intervals (CIs). A clinically important finding in the breast was considered to be a cancer.

\section{Results \\ Breast findings}

440 body MRI examinations of 440 women (mean age: $57 \pm 20$ years) included breast tissue in the field-of-view (FOV range in abdominal MRI in millimetres: $340 \times 276$ to $400 \times 337$; FOV range in chest MRI: $350 \times 295$ to $380 \times 344$ ). Among the 440 body MRI examinations, 302 body MRI were performed on a $1.5 \mathrm{~T}$ scanner and 138 body MRI were performed on a $3.0 \mathrm{~T}$ scanner. In 201 patients (45.7\%), MRI showed more than $50 \%$ of breast tissue, whereas in 239 patients (54.3\%) showed less than $50 \%$ of breast tissue. MRI indications were: $\mathrm{N}=216$ liver imaging, $\mathrm{N}=68$ pancreatic imaging, $\mathrm{N}=32$ renal imaging, $\mathrm{N}=17$ adrenal imaging and $\mathrm{N}=14 \mathrm{MR}$ enterography; $\mathrm{N}=66$ mediastinum and $\mathrm{N}=27$ thoracic wall imaging. In 286 patients, body MRI was performed with intravenous contrast material injection; in 145 patients was performed gadoxetic acid enhanced MRI, in 109 patients was performed gadobenate dimeglumineenhanced and in 32 patients gadoteridolo enhanced MRI.

Breast findings were identified in 41 of 440 patients $(9.3 \%)$. All these patients had a single breast finding. Table 1 shows body MRI with breast finding by BI-RADS category. Of the 41 patients with breast findings detected at body MRI, 25 patients (59.5\%) were categorized as BI-RADS 2; among these, three patients (12.0\%) were downgraded from an initial BI-RADS 3 or 4 by previous imaging work-up or review of the electronic medical record. Among the 25 findings classified as BI-RADS 2, the most common included simple breast cysts [88.0\% (22 of 25)]; previously known round or oval smoothly marginated hypervascular masses with some fat inside were the other benign findings [12.0\% (3 of 25)].

A total of 16 breast incidental findings were categorized as BI-RADS 3 and 4, and the most common imaging findings included breast enhancing masses detected after contrast injection [87.5\% (14 of 16)]. The others imaging finding of BI-RADS 3 and 4 are described in Table 1.

Among the 41 patients with breast findings, 34 patients [82.9\% (34 of 41)] had breast finding identified during abdominal MRI and seven patients [17.1\% (7 of 41)] had breast finding identified during chest MRI (Table 2). Examples of patients with breast findings are shown in Figs. 2, 3 and 4.

Of the 347 patients who underwent abdominal MRI, 34 patients $(9.8 \%)$ had a breast finding and of the 93 patients who underwent chest MRI, seven patients (7.5\%) had a breast finding $(\mathrm{p}=0.69)$.

\section{Further imaging recommendation and clinical relevance}

Of the 440 body MRI examinations, 16 patients (3.6\% [95\% CI $1.6 \%, 5.6 \%$ ]) had a recommendation for an additional imaging to be performed for further evaluation of a breast finding. Of these 16 patients, 13 patients (81\%) were evaluated as BI-RADS category 3 and three patients (19\%) were evaluated as BI-RADS 4. Patient with BI-RADS 2 findings did no receive recommendation for additional imaging. Only one patient with chest MRI received additional imaging recommendation for a BI-RADS 3 finding.

Of the 347 patients who underwent abdominal MRI, 15 patients $(4.3 \%)$ received additional recommendation for further imaging and procedures. Of the 93 patients who underwent chest MRI, one patient $(1.1 \%)$ received additional recommendation for further imaging for a BIRADS 3 breast finding $(\mathrm{p}=0.212)$.

Patients with contrast-enhanced MRI were significantly more likely to have a recommendation for additional 
Table 1 Body MRI findings classified using BI-RADS category with corresponding MRI characteristics

\begin{tabular}{llcl}
\hline BI-RADS category (0-4) & $\begin{array}{l}\text { Number of patient with } \\
\text { breast incidental findings }\end{array}$ & $\begin{array}{l}\text { Breast incidental } \\
\text { finding (\%) }\end{array}$ & MRI characteristic \\
\hline BI-RADS 2 + 3+4 & 41 & 100 & $\begin{array}{c}\text { Rounded lesion hyperintense on } \\
\text { T2-weighted or STIR sequences } \\
\text { BI-RADS 2 }\end{array}$ \\
& 25 & 59.5 & $\begin{array}{c}\text { Rounded/oval circumscribed mass } \\
\text { previously described or evaluated }\end{array}$ \\
BI-RADS 3 & 3 & 12.0 & Rounded/oval circumscribed mass not \\
& 13 & 30.9 & $\begin{array}{c}\text { Oreviously described or evaluated } \\
\text { oval mass }\end{array}$ \\
& 8 & 61.5 & $\begin{array}{c}\text { T2-weighted or STIR hyperintense } \\
\text { irregular lesion }\end{array}$ \\
BI-RADS 4 & 4 & 30.8 & Suspicious enhancing mass \\
& 1 & 7.7 & Architectural distortion
\end{tabular}

The MRI characteristics represent the imaging characteristics of the incidental breast finding detected on body MRI examinations included in the study

Table 2 Breast incidental finding in abdominal and chest MRI examinations

\begin{tabular}{|c|c|c|c|c|c|c|}
\hline \multirow{2}{*}{$\begin{array}{l}\text { Incidental finding by BI- } \\
\text { RADS category }\end{array}$} & \multicolumn{3}{|l|}{ Chest MRI $(\mathrm{N}=93)$} & \multicolumn{3}{|c|}{ Abdominal MRI $(\mathrm{N}=347)$} \\
\hline & $\begin{array}{l}\text { Contrast-enhanced } \\
\text { MRI }\end{array}$ & $\begin{array}{l}\text { Non-enhanced } \\
\text { MRI }\end{array}$ & Total (\%) & $\begin{array}{l}\text { Contrast-enhanced } \\
\text { MRI }\end{array}$ & $\begin{array}{l}\text { Non-enhanced } \\
\text { MRI }\end{array}$ & Total (\%) \\
\hline Total & 5 & 2 & $7(7.5 \%)$ & 19 & 15 & $34(9.8 \%)$ \\
\hline BI-RADS 2 & 4 & 2 & $6(6.4 \%)$ & 4 & 14 & $18(5.2 \%)$ \\
\hline BI-RADS 3 & 1 & $\mathrm{Nf}$ & $1(1.1 \%)$ & 12 & $\mathrm{Nf}$ & $12(3.5 \%)$ \\
\hline BI-RADS 4 & $\mathrm{Nf}$ & $\mathrm{Nf}$ & $\mathrm{Nf}$ & 2 & 1 & $3(0.9 \%)$ \\
\hline
\end{tabular}

Body (chest and abdominal) MRI included in the study $N=440$

$n f$ not found

imaging evaluation compared with patients undergoing non contrast-enhanced MRI (non-enhanced MRI, one patients; contrast-enhanced MRI, 15 patients; $p=0.015$ ).

All patients who underwent contrast-enhanced MRI were, on average, aged 63.5 years (age range, $19-84$ years) compared with 59.9 years (age range, 26-88 years) for all patients with non contrast-enhanced MRI ( $\mathrm{p}=0.0063)$.

All patients who underwent abdominal MRI were, on average, aged 61.8 years (age range, $19-86$ years) compared with 51.2 years (age range, 23-88 years) for all patients with chest MRI ( $\mathrm{p}<0.0001)$.

Overall, $3.6 \%$ (16 of 440) of female patients with breast tissue on body MRI received a recommended additional imaging examinations for further evaluation of a probably benign (BI-RADS 3) or suspicious (BI-RADS 4) breast finding by using body MRI. Of the 14 patients [34.1 \% (14 of 41)] with breast finding who underwent the recommended additional imaging on the basis of the body MRI findings, 11 patients $(78.6 \%)$ were BI-RADS 3 and three patients $(21.4 \%)$ were BI-RADS 4 . Two patients [12.5\%
(2 of 16)] with BI-RADS 3 were lost at follow-up and did not undergo the additional imaging recommended. No additional imaging was recommended for incidental breast findings categorized as BI-RADS 2.

Clinical relevance of breast incidental finding was available for 14 patients (follow-up interval range, 6-14 months). After undergoing additional imaging examinations, three patients [21.4\% (3 of 14)] had a clinically important finding. Two patients had a previously unknown invasive breast cancer (invasive ductal carcinoma) and one patient had a previously known invasive breast cancer (invasive ductal carcinoma) (Table 3). Therefore, $18.7 \%$ (3 of 16) of women undergoing further imaging work-up because of recommendation of breast incidental finding with body MRI had a clinically important finding in the breast.

Three of 440 patients $(0.7 \%)$ had a clinically important finding identified with body MRI, and these included two patients who had previously unknown invasive breast cancer. 


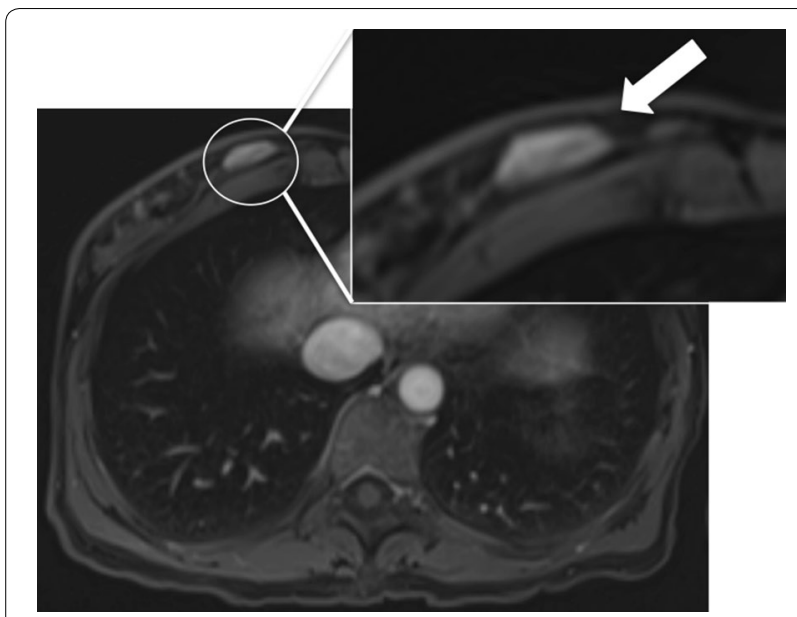

Fig. 2 Examples of breast findings by BI-RADS category. A 49-yearwoman underwent abdominal MRI for liver evaluation. Threedimensional dynamic axial volumetric interpolated breath-hold examination image after intravenous power injection of $0.025 \mathrm{mmol} /$ $\mathrm{kg}$ of gadoxetic acid (Primovist) at $60 \mathrm{~s}$. Less than $50 \%$ of the breast tissue was visible at abdominal MRI. An oval nodule (white circle) was found in the inner quadrant of the right breast $(20 \mathrm{~mm})$. The breast finding was first categorized as BI-RADS RM 3. After electronic medical record review this finding was downgraded as BI-RADS RM 2 (known fibrolipoma). In the upper right corner is highlighted the breast findings (white arrow)

One patient with a clinically important finding had an indication for liver MRI. One patient had an indication for pancreatic imaging and the other patient had an indication for MR cholangiography. One of the three patients with a malignant breast lesion had a previous history of breast cancer. The remaining two patients had no previous history of malignancies (Table 3).

\section{Cost of further imaging and procedures}

Of the 16 patients with recommendations for additional imaging, four patients had one additional imaging study recommended, and four patients had two additional imaging studies recommended, one patient had three additional imaging recommendation, five patients had one additional imaging and one procedures recommendation, and two patient had two additional imaging and two procedures recommendations, which totalled 33 additional imaging studies and procedures that were recommended (Table 4). Enhancing mass accounted for $94 \%$ (31 of 33) of these recommendations, including 19 of the 21 recommendations for breast ultrasonographic imaging. Of the imaging studies that were performed, ultrasound examinations were the majority [61 \% (19 of 31$)$ ] of additional imaging examinations recommended for diagnosis of newly identified breast findings.

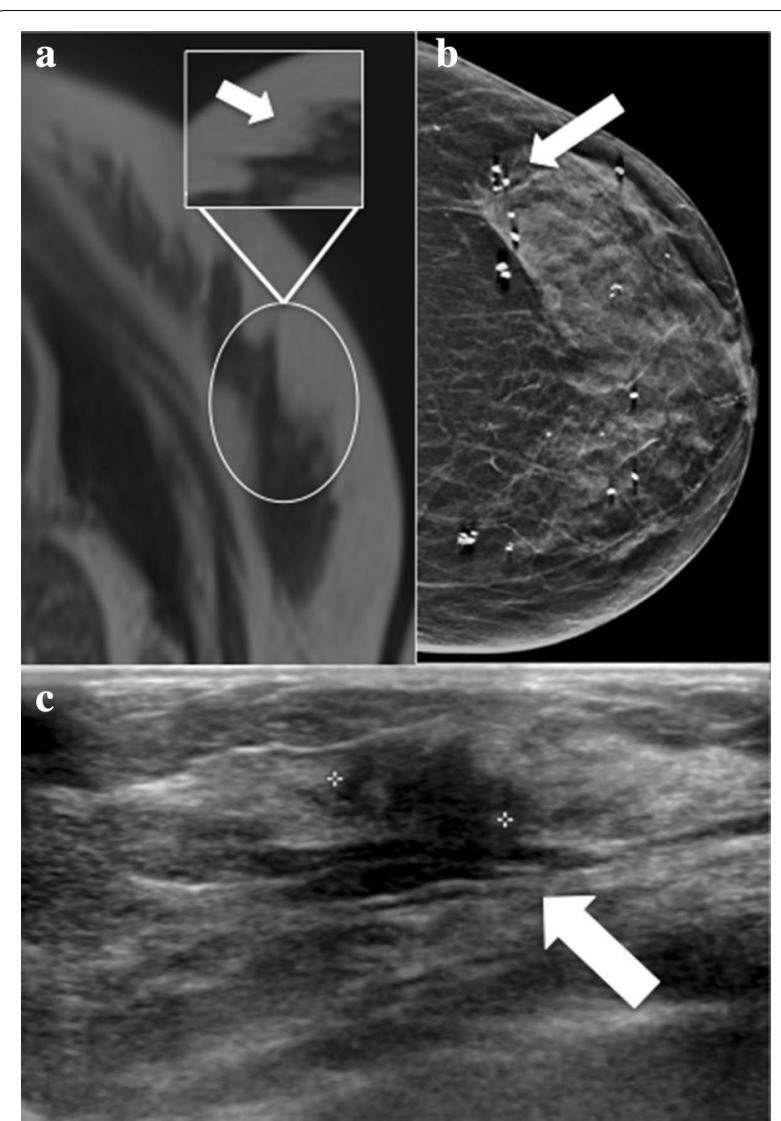

Fig. 3 Examples of breast incidental findings by BI-RADS category. a A 75-year-woman (Patient 1 in Table 3) underwent MR cholangiopancreatography. More than $50 \%$ of the breast tissue was visible at abdominal MRI. A suspicious architectural distortion (white circle) was found in the outer quadrants of the left breast (BI-RADS RM 4). In the box is highlighted the breast findings. b Left full-filed digital mammography shows an area of architectural distortion (white arrow) in the outer quadrants. c Corresponding US image shows a mass with indistinct borders in the left breast at 2 o'clock position $(15 \mathrm{~mm}$ diameter). The US-guided core needle biopsy confirmed a B5b lesion (invasive ductal carcinoma)

Subsequent imaging studies and additional costs resulted are shown in Table 4.

The total costs for all subsequent imaging studies and procedures performed as a result of a body MRI recommendation for additional imaging evaluation and procedures was $€ 4440$. The entire procedures constituted the $9 \%$ ( $€ 400$ of $€ 4440)$ of the additional costs (Table 4). The 2015 costs at our Institution of contrast-enhanced abdominal or chest MRI examination is $€ 450$, non contrast-enhanced is $€ 350$. Therefore, for each of the 440 body MRI examinations included in this study an average additional cost of $€ 10.1$ was attributable to the additional imaging recommended. 


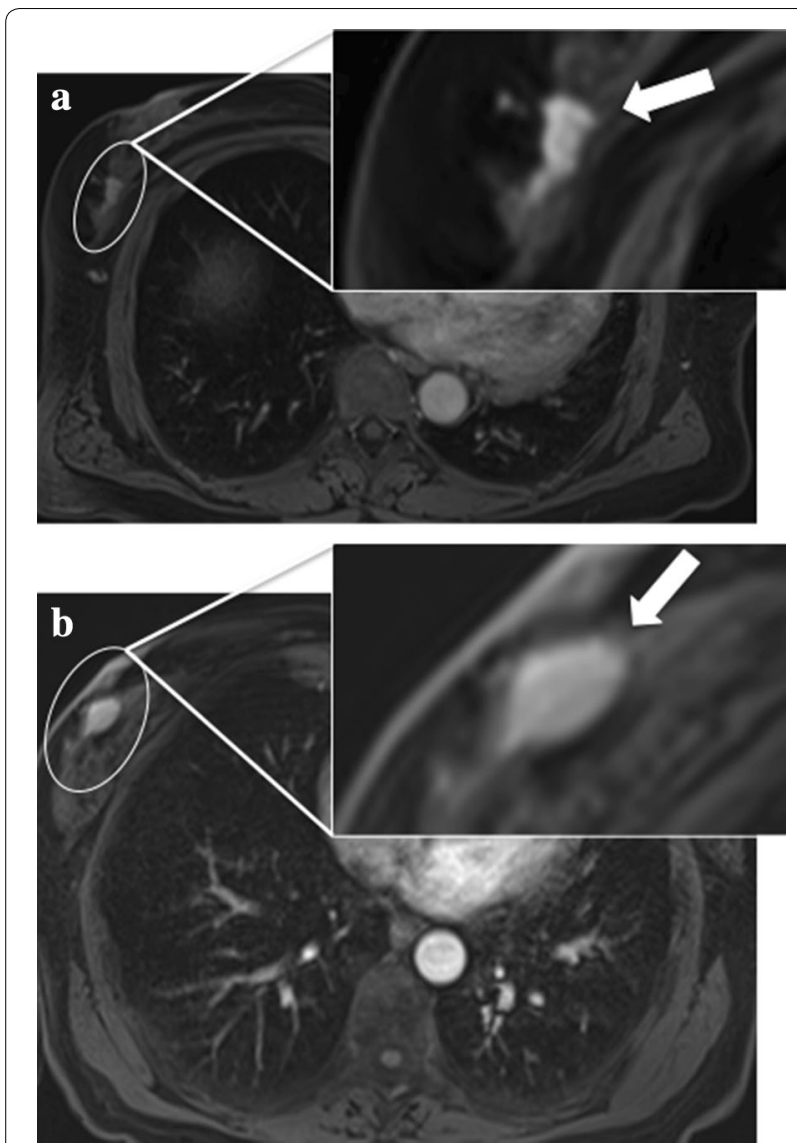

Fig. 4 Examples of breast incidental findings by BI-RADS category. a A 67-year-woman (Patient 2 in Table 3) underwent abdominal MRI for follow-up of a cystic lesion of the head of the pancreas. Threedimensional dynamic axial volumetric interpolated breath-hold examination image after intravenous power injection of $0.05 \mathrm{mmol} /$ $\mathrm{kg}$ of Gadobenate dimeglumine (Multihance) at $60 \mathrm{~s}$. More than $50 \%$ of the breast tissue was visible at abdominal MRI. A suspicious mass (white circle) was found in the outer quadrants of the right breast (BI-RADS RM 4) The US-guided core needle biopsy confirmed a B5b lesion (Invasive Ductal Carcinoma). In the upper right corner is highlighted the breast findings (white arrow). b A 58-year-woman (Patient 3 in Table 3) underwent abdominal MRI for liver evaluation. Three-dimensional dynamic axial volumetric interpolated breath-hold examination image after intravenous power injection of $0.025 \mathrm{mmol} /$ $\mathrm{kg}$ of gadoxetic acid (Primovist) at $60 \mathrm{~s}$. More than $50 \%$ of the breast tissue was visible at abdominal MRI. A suspicious mass (white circle) was found in the outer quadrants of the right breast (BI-RADS RM 4). This patient had a previously known breast cancer in the outer quadrants of the right breast, corresponding to the breast finding detected on MR images. In the upper right corner is highlighted the breast findings (white arrow)

Sub-analyses of cost were performed for the 16 of 41 body MRI examinations with breast findings that resulted in recommendations for additional imaging evaluation. The 2015 costs at our Institution of contrastenhanced abdominal or chest MRI examination is $€ 450$, non contrast-enhanced is $€ 350$. Therefore, for each of the 41 body MRI with incidental breast finding performed in this study, an average additional cost of $€ 108.3$ was attributable to the additional imaging recommended.

\section{Discussion}

Breast cancer is the most frequent cancer in women and is the leading cause of cancer-related deaths of European women (Senkus et al. 2015; Sardanelli et al. 2010; Mann et al. 2015). Breast MRI is a highly sensitive imaging method for breast cancer detection (Senkus et al. 2015; Sardanelli et al. 2010; Mann et al. 2015; Morrow et al. 2011). For breast cancer screening, abbreviated and nonstandard MRI protocols have been evaluated (Kuhl et al. 2014; Mango et al. 2015; Carbonaro et al. 2012).

In clinical practice, a significant amount of breast tissue is commonly visible during body MRI. Therefore, given the high sensitivity of MRI, there could be some breast findings visible in the field-of-view. In this study, we aimed to assess breast findings in women undergoing body MRI. To the best of our knowledge, this is the first study assessing the presence of breast lesions incidentally detected on abdominal and chest MRI. We found that, among female patients undergoing body MRI, at least $3.6 \%$ had a recommendation for additional imaging examinations and procedures due to an incidental breast finding, and $0.7 \%$ had a clinically relevant incidental breast finding. Notably, we found three invasive breast cancers (invasive ductal carcinoma). One patient undergoing non contrast-enhanced abdominal MRI had an incidental breast finding that received recommendation for further imaging work-up and this patient was revealed to have a breast cancer. The relative high prevalence of breast cancer in the group of patients undergoing non contrast-enhanced body MRI compared to the group of patients undergoing contrast-enhanced MRI could be related to the high intrinsic sensitivity of contrast-enhanced body MRI that leads to a higher detection of breast findings compared to the other group. For the same reason, the majority of breast findings detected during contrast-enhanced MRI had higher BI-RADS category compared to the breast findings detected during non-contrast-enhanced MRI. However, this consideration has to be confirmed on larger series. The European Society of Breast Imaging (EUSOBI) guidelines acknowledge that breast MRI should be performed routinely with contrast agent for diagnostic value (Mann et al. 2015), indeed, we do not believe that non contrast-enhanced body MRI could be an accurate imaging modality for breast cancer detection. In addition, our results do not support the use of body MRI as an additional screening modality for breast cancer detection, but highlight the possibility to find a breast lesion on body MRI. 
Table 3 Patients with clinical relevant breast finding

\begin{tabular}{|c|c|c|c|c|c|c|c|c|}
\hline Patient & Age (years) & $\begin{array}{l}\text { Previous history } \\
\text { of cancer }\end{array}$ & MRI performed & $\begin{array}{l}\text { Field of } \\
\text { view }\end{array}$ & $\begin{array}{l}\text { Contrast } \\
\text { media }\end{array}$ & $\begin{array}{l}\text { Clinical indica- } \\
\text { tion }\end{array}$ & $\begin{array}{l}\text { BI-RADS } \\
\text { category of the } \\
\text { incidental find- } \\
\text { ing at body MRI }\end{array}$ & $\begin{array}{l}\text { Final diagnosis } \\
\text { at surgery }\end{array}$ \\
\hline 1 & 75 & $\begin{array}{l}\text { Previous history } \\
\text { of breast } \\
\text { cancer }\end{array}$ & Abdominal MRI & $380 \times 261$ & Not used & $\begin{array}{l}\text { MR cholangio- } \\
\text { graphy }\end{array}$ & BI-RADS 4 & $\begin{array}{l}\text { Invasive ductal } \\
\text { carcinoma }\end{array}$ \\
\hline 2 & 67 & No history & Abdominal MRI & $380 \times 380$ & $\begin{array}{l}\text { Gadobenate } \\
\text { dimeglumine }\end{array}$ & Pancreatic MRI & BI-RADS 4 & $\begin{array}{l}\text { Invasive ductal } \\
\text { carcinoma }\end{array}$ \\
\hline 3 & 58 & No History & Abdominal MRI & $308 \times 380$ & Gadoxetic acid & Liver MRI & BI-RADS 4 & $\begin{array}{l}\text { Invasive ductal } \\
\text { carcinoma }\end{array}$ \\
\hline
\end{tabular}

Table 4 Additional imaging studies and work-up recommended and performed because of breast findings at body MRI and associated additional costs

\begin{tabular}{llllll}
\hline & FFDM & US & $\begin{array}{l}\text { US core-needle } \\
\text { biopsy }\end{array}$ & $\begin{array}{l}\text { Ultrasound-guided } \\
\text { preoperative lesion } \\
\text { marking }\end{array}$ & $\begin{array}{l}\text { Number of additional } \\
\text { imaging studies and } \\
\text { work-up recommended }\end{array}$ \\
\hline $\begin{array}{l}\text { Chest MRI } \\
\text { Abdominal MRI }\end{array}$ & 3 & 2 & & 2 & 2 \\
imaging studies and \\
wotal
\end{tabular}

FFDM full-field digital mammography, US ultrasound

Two ultrasound examinations were not performed (patients lost at follow-up)

The prevalence of our findings is consistent with other cross-sectional imaging examinations: the detection of clinically relevant incidental breast findings varies from $0.3 \%$ on computed tomography of the body (Monzawa et al. 2013), to $6 \%$ on PET-CT (Benveniste et al. 2015). Regarding previous studies dealing with incidental breast finding on MRI, it has been evaluated the prevalence of breast cysts in whole-body MRI, which was $3.5 \%$ for patients over the age of 50 years (Cieszanowski et al. 2014). However, breast cysts are not clinically relevant. A recent report (Bamberg et al. 2015) shows the design of the MRI Study of the German National Cohort with a particular mention about incidental findings: a major challenge is that the whole-body unenhanced MR protocol is different from that used on diagnostic application, and, for examples, breast cancer cannot be detected with reasonable specificity. Differently from previous studies, we reviewed both non-contrast and contrast-enhanced abdominal and chest MRI examinations and we were able to detect not only breast cysts but more suspicious breast incidental findings too.

Regarding the percentage of breast tissue visible in our study population during MRI, the majority of patients had less than $50 \%$ of breast tissue visible. Therefore, it is possible that other breast findings would have been visible using a larger FOV. Although this study is formally retrospective, the MR images review allowed a simileprospective reading of all images with a blind assessment of the breast tissue that would have not been feasible with the review of the MR report alone. This technique of reading was very time consuming, but we believe that increase and strengthen the results of the study. Regarding costs, we found that additional imaging and procedures for breast findings contributed an increased cost of $€ 108.3$ for each patient with incidental findings. However, additional recommendations for breast finding do not increase cost significantly per women undergoing body MRI with breast tissue visible; indeed, an average additional cost of $€ 10.61$ was totalled for each patient included in the study. We acknowledge that the costs of additional recommendation lacks of the costs of the surgical procedures performed for breast cancer therapy, underestimating the average additional costs per patient. On the contrary, we could not perform a cost-analysis about the saving costs for early breast cancer detection.

Our study has other limitations. First, we did not assess the correlation between detection of breast finding and clinical characteristic and technical parameters. In addition, we did not evaluate the histological size of the breast cancers because the evaluation of the incidence of breast cancers during body MRI and its correlation with breast cancer characteristics was not the purpose of our study. Finally, the study design could have underestimated the number of total breast findings due to inclusion of patients with percentage of breast tissue visible and not the whole breast. 


\section{Conclusions}

In conclusion, at least $3.6 \%$ of women undergoing body MRI had a breast incidental finding, and $18.7 \%$ of these patients had a clinically relevant incidental breast finding. Further imaging work-up and procedures recommended did not increase significantly the cost per women undergoing body MRI.

\begin{abstract}
Abbreviations
MRI: magnetic resonance imaging; CT: computed tomography; FOV: field-ofview; FDG PET/CT: (18) F-fluorodeoxyglucose positron emission tomographycomputed tomography; Cls: confidence intervals; BI-RADS: breast imaging reporting and data system; EUSOBI: European Society of Breast Imaging.
\end{abstract}

\section{Authors' contributions}

$\mathrm{BB}$ and $\mathrm{AT}$ contributed to planning the study, the analysis, interpretation of the data and drafting of the article. BB, GS, FN, MP, LG, SB, LS and MC contributed to the data collection. $\mathrm{BB}, \mathrm{MC}$ and $\mathrm{AT}$ contributed to study conception. All authors read and approved the final manuscript.

\section{Author details}

${ }^{1}$ Department of Health Sciences (DISSAL), University of Genova, Via A. Pastore 1, 16132 Genoa, Italy. ${ }^{2}$ Department of Diagnostic Senology, IRCCS Azienda Ospedaliera Universitaria San Martino IST-Istituto Nazionale per la Ricerca sul Cancro, Largo Rosanna Benzi 10, 16132 Genoa, Italy. ${ }^{3}$ Institute of Anatomy, Department of Experimental Medicine, University of Genova, Via Leon Battista Alberti, 2, 16132 Genoa, Italy

\section{Competing interests}

The authors declare that they have no competing interests.

\section{Availability of data and materials}

The dataset supporting the conclusions of this article is included within the article itself.

\section{Statement of informed consent}

This study was notified to the Institutional Review Board, and the requirement for informed consent was waived.

Received: 11 May 2016 Accepted: 12 May 2016

Published online: 18 June 2016

\section{References}

American college of radiology breast imaging reporting and data system (BIRADS) Atlas (2013), 5th edn. Reston, VA; 2013

Bach AG, Abbas J, Jasaabuu C, Schramm D, Wienke A, Surov A (2013) Comparison between incidental malignant and benign breast lesions detected by computed tomography: a systematic review. J Med Imaging Radiat Oncol 57:529-533

Bamberg F, Kauczor HU, Weckbach S et al (2015) Whole-body MR imaging in the German National Cohort: rationale, design, and technical background. Radiology 277:206-220

Bazzocchi A, Spinnato P, Garzillo G et al (2012) Detection of incidental vertebral fractures in breast imaging: the potential role of MR localisers. Eur Radiol 22:2617-2623

Benveniste AP, Marom EM, Benveniste MF, Mawlawi O, Fox PS, Yang W (2015) Incidental primary breast cancer detected on PET-CT. Breast Cancer Res Treat 151:261-268

Carbonaro LA, Tannaphai P, Trimboli RM, Verardi N, Fedeli MP, Sardanelli F (2012) Contrast enhanced breast MRI: spatial displacement from prone to supine patient's position. Preliminary results. Eur J Radiol 81:771-774

Chang KJ, Kamel IR, Macura KJ, Bluemke DA (2008) 3.0-T MR imaging of the abdomen: comparison with 1.5 T. Radiographics 28:1983-1998
Chernyak V, Flusberg M, Haramati LB, Rozenblit AM, Bellin E (2015) Incidental pancreatic cystic lesions: is there a relationship with the development of pancreatic adenocarcinoma and all-cause mortality? Radiology 274:161-169

Cieszanowski A, Maj E, Kulisiewicz P et al (2014) Non-contrast-enhanced whole-body magnetic resonance imaging in the general population: the incidence of abnormal findings in patients 50 years old and younger compared to older subjects. PLOS ONE 9:e107840

Dewey M, Schnapauff D, Teige F et al (2007) Non-cardiac findings on coronary computed tomography and magnetic resonance imaging. Eur Radiol 17:2038-2043

Erturk SM, Alberich-Bayarri A, Herrmann KA, Marti-Bonmati L, Ros PR (2009) Use of 3.0-T MR imaging for evaluation of the abdomen. Radiographics 29:1547-1563

Heller MT, Harisinghani M, Neitlich JD, Yeghiayan P, Berland LL (2013) Managing incidental findings on abdominal and pelvic CT and MRI, part 3: white paper of the ACR Incidental Findings Committee II on splenic and nodal findings. J Am Coll Radiol 10:833-839

Khosa F, Krinsky G, Macari M, Yucel EK, Berland LL (2013) Managing incidental findings on abdominal and pelvic CT and MRI, Part 2: white paper of the ACR Incidental Findings Committee II on vascular findings. J Am Coll Radiol 10:789-794

Kuhl CK, Schrading S, Strobel K, Schild HH, Hilgers RD, Bieling HB (2014) Abbreviated breast magnetic resonance imaging (MRI): first postcontrast subtracted images and maximum-intensity projection-a novel approach to breast cancer screening with MRI. J Clin Oncol 232:2304-2310

Lee CU, White DB, Sykes AM (2014) Establishing a chest MRI practice and its clinical applications: our insight and protocols. J Clin Imaging Sci 4:17

Mango VL, Morris EA, David Dershaw D et al (2015) Abbreviated protocol for breast MRI: are multiple sequences needed for cancer detection? Eur J Radiol 84:65-70

Mann RM, Balleyguier C, Baltzer PA et al (2015) Breast MRI: EUSOBI recommendations for women's information. Eur Radiol 25:3669-3678

Maxwell AW, Keating DP, Nickerson JP (2015) Incidental abdominopelvic findings on expanded field-of-view lumbar spinal MRI: frequency, clinical importance, and concordance in interpretation by neuroimaging and body imaging radiologists. Clin Radiol 70:161-167

Monzawa S, Washio T, Yasuoka R, Mitsuo M, Kadotani Y, Hanioka K (2013) Incidental detection of clinically unexpected breast lesions by computed tomography. Acta Radiol 54:374-379

Morrow M, Waters J, Morris E (2011) MRI for breast cancer screening, diagnosis, and treatment. Lancet 378:1804-1811

Niell BL, Bennett D, Sharma A, Gazelle GS (2015) Extramammary findings on breast mr examinations: frequency, clinical relevance, and patient outcomes. Radiology 276:56-64

Patel MD, Ascher SM, Paspulati RM et al (2013) Managing incidental findings on abdominal and pelvic CT and MRI, part 1: white paper of the ACR Incidental Findings Committee II on adnexal findings. J Am Coll Radiol 10:675-681

Sardanelli F, Boetes C, Borisch B et al (2010) Magnetic resonance imaging of the breast: recommendations from the EUSOMA working group. Eur J Cancer 46:1296-1316

Schmidt CO, Hegenscheid K, Erdmann P et al (2013) Psychosocial consequences and severity of disclosed incidental findings from whole-body MRI in a general population study. Eur Radiol 23:1343-1351

Sebastian S, Araujo C, Neitlich JD, Berland LL (2013) Managing incidental findings on abdominal and pelvic CT and MRI, Part 4: white paper of the ACR Incidental Findings Committee II on gallbladder and biliary findings. J Am Coll Radiol 10:953-956

Senkus E, Kyriakides S, Ohno S et al (2015) Primary breast cancer: ESMO clinical practice guidelines for diagnosis, treatment and follow-up. ESMO Guidelines Committee Ann Oncol 26(Suppl 5):v8-v30

Tarnoki DL, Tarnoki AD, Richter A, Karlinger K, Berczi V, Pickuth D (2015) Clinical value of whole-body magnetic resonance imaging in health screening of general adult population. Radiol Oncol 49:10-16

Wagner J, Aron DC (2012) Incidentalomas: a "disease" of modern imaging technology. Best Pract Res Clin Endocrinol Metab 26:3-8

Wyttenbach R, Medioni N, Santini P et al (2012) Extracardiac findings detected by cardiac magnetic resonance imaging. Eur Radiol 22:1295-1302 